\title{
Investigation Risk Factors of Cardiovascular Disease in Khartoum State, Sudan: Case - Control Study 2015
}

\author{
Ehab A. M. Frah ${ }^{1}$, Mohammed A. M. Naeem ${ }^{2}$ \\ ${ }^{1}$ University of Bahri, College of Economic and Social Studies, Sudan \\ Currently University of Taluk, faculty of Science, Saudi Arabia \\ ${ }^{2}$ Nile Valley University, Postgraduate College
}

\begin{abstract}
Background: Cardiovascular diseases (CVD) are a foremost cause of morbidity and mortality in Sudan and global with the rapid increasing prevalence, due to urbanization and changes in lifestyle. Objective: To investigate the cardiovascular disease risk factors among Sudanese who live in Khartoum state. Methods: A case-control study was conducted in the Ahmed Gasim HospitalCardiac Surgery and renal Transplantation Center targeting the cardiovascular disease and were included patients who came for treatment or follow-up during 2015. A total of 162 patients with cardiovascular Disease (CVD) in Ahmed Gasim Hospital- Cardiac Surgery and renal Transplantation Center and 162 control who are not patients or have any history of cardiovascular diseases. They were interviewed using purposively designed questionnaires. Logistic regression has been used for modeling the probability that a cardiovascular Disease (CVD) could be developed as a function of risk factors. Results: This study shows that high bold pressure, family history and lack of physical activity are the main risk factors of cardiovascular diseases in Khartoum states, Sudan. Conclusion: Hypertension, family history of CVD and life style were found to be main risk factors of CVD.
\end{abstract}

Keywords: cardiovascular, risk factors, logistic regression

\section{Introduction}

Cardiovascular diseases are the leading cause of death globally. This is true in all areas of the world (W.H.O, 2011).Together they resulted in 17.3 million deaths (31.5\%) in 2013 up from 12.3 million (25.8\%) in 1990.CVD is responsible for $30 \%$ of all deaths in the world (W.H.O, 2009). In Africa; the rate is increasing rapidly and it is now a public health concern. It has a major socio-economic impact on individuals, families and societies in terms of healthcare costs, work absenteeism and national productivity. The most important CVD in the African region are hypertension, atherosclerosis, cardiomyopathies and rheumatic heart disease. (Opieet al, 2005, W.H.O, 2011).

Until the fifties coronary heart disease (CHD) was rare among Sudanese. The study of Halim in 1961 reported ischemic heart disease as accounting up to $12.6 \%$ of Khartoum Hospital admissions (Halimet al, 1961). The disease has since shown increasing rates and by 1984 it formed $17.2 \%($ Khalil et al, 1984).

A recent (2011) hospital based study of cardiovascular admission was done in Shaab Hospital, Khartoum. The pattern of heart disease in the same study was reported CHD reaching65\% among all admissions (Suliman et al, 1984).These patterns have reflected as high prevalence of heart failure rates of $73 \%$ in acute admissions at $\mathrm{Al}$ Shaba Hospital. A recent study of heart failure in Sudan reported ischemic cardiomyopathy as the leading cause of heart failure in Sudan. (Khalil et al, 2012).

\section{Cardiovascular Risk Factors}

The majority of CVD is caused by risk factors that can be controlled treated or modified, such as high blood pressure, cholesterol, overweight/obesity, tobacco use, lack of physical activity and diabetes. However, there are also some major CVD risk factors that cannot be controlled or Non-modifiable risk factor as age, gender and family history. In terms of attributable deaths, the leading cardiovascular disease risk factor is raised blood pressure (to which 13\% of global deaths is attributed), followed by tobacco use $9 \%$, raised blood glucose $6 \%$, physical inactivity $6 \%$ and overweight and obesity $5 \%$.( W.H.O., 2011).

\section{Firstly: Modifiable Risk Factors}

Hypertension (high blood pressure): Blood pressure is measured as two numbers, written one over the other and recorded in millimeters of mercury. Tobacco use: Smoking is estimated to cause nearly 10 per cent of all CVD. Raised blood glucose (diabetes):Diabetes is defined as having a fasting plasma glucose value of 7.0 $\mathrm{mmol} / \mathrm{l}(126 \mathrm{mg} / \mathrm{dl})$ or higher. Physical inactivity: Insufficient physical activity can be defined as less than five times 30 minutes of moderate activity per week, or less than three times 20 minutes of vigorous activity per week, or equivalent. Unhealthy diet: High dietary intakes of saturated fat, trans-fats and salt and low intake of fruits, vegetables and fish are linked to CVD. Cholesterol/lipids: Raised blood cholesterol increases the risk of heart disease and stroke. Globally, one third of is chaemic heart disease is attributable to high cholesterol. Overall, raised cholesterol is estimated to cause 2.6 million deaths (4.5 per cent of total) and 29.7 million disability-adjusted life year (DALYs), or 2 per cent of total DALYS globally. Overweight and obesity: Obesity is strongly related to major cardiovascular risk factors such as raised blood pressure, glucose intolerance, type 2 diabetes and dyslipidaemia. Worldwide, at least 2.8 million people die each year as a result of being overweight or obese, and an 


\section{International Journal of Science and Research (IJSR) \\ ISSN (Online): 2319-7064 \\ Index Copernicus Value (2013): 6.14 | Impact Factor (2014): 5.611}

estimated 35.8 million (2.3 per cent) of global DALYs are caused by overweight or obesity.( W.H.O, 2011).

\section{Secondly: Non-Modifiable Risk Factors}

Age: CVD becomes increasingly common with advancing age. As a person gets older, the heart undergoes subtle physiologic changes, even in the absence of disease. Gender: A man is at greater risk of heart disease than a pre-menopausal woman. Once past the menopause, a woman's risk is similar to a man's. Risk of stroke, however, is similar for men and women. Family history: A family's history of CVD indicates a person's risk. If a first-degree blood relative has had coronary heart disease or stroke before the age of 55 years (for a male relative) or 65 years (for a female relative), the risk increases.(W.H.O, 2011).

\section{Patient and Methodology}

A case-control study conducted in the Ahmed Gasim Hospital- Cardiac Surgery and renal Transplantation Center targeting the cardiovascular disease patients who came for treatment or follow-up during 2015A total of 162 patients with cardiovascular Disease (CVD) in Ahmed Gasim Hospital- Cardiac Surgery and renal
Transplantation Center and 162 control who are not patients or have any history of cardiovascular diseases. They were interviewed using purposively designed questionnaires. All cardiovascular disease patients cases registered in Ahmed Gasim Hospital- Cardiac Surgery and renal Transplantation Center in the period from 2010 to 2015 were also analyzed.

Logistic regression have been used for modeling the probability that a cardiovascular disease risk factors could be developed as a function of high blood pressure, cholesterol, overweight/obesity, tobacco use, lack of physical activity, diabetes, age, gender and family history. Logistic regression methods were used to fit with the multiple logistic regression models, testing for the significance of the coefficients and the confidence interval estimation. The goal of any method used was to select those variables resulted in best model within the scientific based context of the problem, seeking for the best model that seems to be containing all those variables (Hosmer and Lemeshow, 2000). In statistical inference procedures we used chi-square distribution based on the likelihood ratio score or Wald test statistics which are good to fit statistics(Hosmer and Lemeshow, 2000; Pampel, 2000).

\section{Data Analysis and Interpretations}

Table 1: Risk factors distribution between case and control

\begin{tabular}{|l|c|c|c|}
\hline \multicolumn{1}{|c|}{ Risk Factor } & Case & Control & P-value \\
\hline Diabetes & $\mathbf{5 2 ( 3 1 . 1 \% )}$ & $\mathbf{3 1 ( 1 9 . 1 \% )}$ & $\mathbf{0 . 0 0 5}$ \\
\hline High Blood Pressure & $\mathbf{9 5}(\mathbf{5 8 . 6 \% )}$ & $\mathbf{1 1 ( 6 . 8 \% )}$ & $\mathbf{0 . 0 0 0}$ \\
\hline Overweight/Obesity & $34(21.4 \%)$ & $30(18.5)$ & 0.33 \\
\hline Cholesterol/Lipids & $13(8 \%)$ & $6(3.7 \%)$ & 0.077 \\
\hline Family History & $\mathbf{7 3 ( 4 5 . 1 \% )}$ & $\mathbf{3 1}(\mathbf{1 9 . 1 \% )}$ & $\mathbf{0 . 0 0 0}$ \\
\hline Unhealthy Diet & $102(63 \%)$ & $97(59.9 \%)$ & 0.324 \\
\hline Lack Of Physical Activity & $\mathbf{9 7 ( 5 9 . 9 \% )}$ & $\mathbf{5 5 ( 3 4 \% )}$ & $\mathbf{0 . 0 0 0}$ \\
\hline Tobacco Use & $\mathbf{3 6 ( 2 2 . 2 )}$ & $\mathbf{2 0 ( 1 2 . 3 )}$ & $\mathbf{0 . 0 1 3}$ \\
\hline
\end{tabular}

As shown in Table (1) the almost risk factors of the studied population are similar to each other and p-values are more than 0.05 so they have the same chance in getting cardiovascular disease. Table (1) shows that the high blood pressures, family history, lack of physical activity and tobacco significant difference since $p$-values are $>0.05$. However, the history of cardiovascular disease risk factors, high blood pressure and lack of physical activity are very high in cases than control.

\section{Logistic Regression}

Logistic regression model was specified and the regression results related to the factors that influence risk factors of cardiovascular disease risk factors were reported.

The results of fitting the analysis of logistic regression model to these data are given in Table (2). This table presents the following information which is given for each variable listed in the first column:
- The estimated slope coefficient for univariate logistic regression model containing only this variable (B).

- The estimated standard error of the estimated slope coefficient (S.E).

- The likelihood ratio test statistic, Wald (G), for the hypothesis that, the slope coefficient is zero. Under the null hypothesis, this quantity follows the chi-square distribution with one degree of freedom (Wald).

- The significance value, level for the likelihood ratio test (Sig.).

- The estimated odds ratio, which was obtained by exponentiation of the estimated coefficient (OR).

Overall percentage: This gives the percent of cases for which the dependent variables were correctly predicted from the model, increased from $50 \%$ for the null model to $77.1 \%$ for the full model.

Hosmer-Lemeshow test: To test null hypothesis there is a linear relationship between the predictor variables and the log odds of the criterion variable. Cases were arranged in order by their predicted probability on the criterion variable and since chi-square statistic is computed 


\section{International Journal of Science and Research (IJSR) \\ ISSN (Online): 2319-7064 \\ Index Copernicus Value (2013): 6.14 | Impact Factor (2014): 5.611}

comparing the observed frequencies with those expected under the linear model. A non significant chi-square indicates that the data fit the model well ( $p$-value $=0.215$ ).

Table 2: Logistic Regression Analysis

\begin{tabular}{|c|c|c|c|c|c|c|c|c|}
\hline & \multirow{3}{*}{$B$} & \multirow{3}{*}{ S.E. } & \multirow{3}{*}{ Wald } & 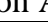 & & & & \\
\hline \multirow{2}{*}{ Risk factors } & & & & \multirow{2}{*}{$d f$} & \multirow{2}{*}{ Sig. } & \multirow{2}{*}{$\operatorname{Exp}(B)$} & \multicolumn{2}{|c|}{ 95\% C.I. for $\operatorname{EXP}(B)$} \\
\hline & & & & & & & Lower & Upper \\
\hline Diabetes & .021 & .356 & .004 & 1 & .952 & .979 & .487 & 1.967 \\
\hline High Blood Pressure & 2.858 & .398 & 51.616 & 1 & .000 & 17.43 & 7.992 & 38.013 \\
\hline Overweight/Obesity & .625 & .407 & 2.364 & 1 & .124 & .535 & .241 & 1.187 \\
\hline Cholesterol/Lipids & .019 & .636 & .001 & 1 & .976 & 1.02 & .293 & 3.542 \\
\hline Family History & .963 & .313 & 9.475 & 1 & .002 & 2.62 & 1.419 & 4.836 \\
\hline Unhealthy Diet & .297 & .298 & .994 & 1 & .319 & 1.35 & .751 & 2.412 \\
\hline Lack Of Physical Activity & .948 & .288 & 10.844 & 1 & .001 & 2.58 & 1.468 & 4.536 \\
\hline Tobacco Use & .095 & .398 & .057 & 1 & .812 & 1.09 & .504 & 2.397 \\
\hline Constant & -1.637 & .334 & 24.046 & 1 & .000 & .195 & & \\
\hline
\end{tabular}

\section{Discussion}

The main purpose of the present study was to examine from an empirical point of view the factors that thought to be risk factors of cardiovascular disease. This was done among two groups; cases of CVD in Ahmed Gasim Hospital- Cardiac Surgery and renal Transplantation Center; and control group form those who live in Khartoum States, Sudan.

To validate that the risk factors of cardiovascular, we introduced logistic regression analysis which is a suitable statistical tool for analysis of this type of data and hypotheses of the research. Logistic regression analysis is an increasingly popular analytic tool usually used to investigate the relationship between a categorical outcome and a set of explanatory variables. The outcome, or response, can be dichotomous (yes, no) or ordinal (low, medium, high). It enables predicting the probability that the "event of interest" will occur as a linear function of one (or more) continuous and/or dichotomous independent variables (Hosmer and Lemeshow, 2000; Pampel, 2000). Logistic regression and least squares regression are almost identical. Both methods produce prediction equations. In both cases, the regression coefficients measure the predictive capability of the independent variables6 (Agresti, 1996).

The results of logistic regression analysis in this study showed that there is a significance difference in risk factors between cases and controls, so the cardiovascular disease risk factors risk factors are the high bold pressure (OR17.43, \pm CI 7.99, 38.02), family history (OR2.62, $\pm \mathrm{CI} 1.42$, 4.84), lack of physical activity (OR 2.58, $\pm \mathrm{CI} 1.47,4.5)$ are the main risk factors.

The limitation of this study is that it is an institution based and of small sample size. Studies that uses statistical analysis are better to be conducted on community basis.

\section{References}

[1] Agresti, A. (1996). An introduction to categorical data analysis. John Wiley and Sons, Inc.

[2] Cardiovascular diseases in the African region: Current situation and perspectives. Report of the Director for the Regional office for Africa. World Health Organization 2009.

[3] Global Atlas on Cardiovascular Disease Prevention and Control. Mendis S, Puska P, Norrving B editors. World Health Organization (in collaboration with the World Heart Federation and World Stroke Organization), Geneva 2011.

[4] Global Atlas on Cardiovascular Disease Prevention and Control. Mendis S, Puska P, Norrving B editors. World Health Organization (in collaboration with the World Heart Federation and World Stroke Organization), Geneva 2011.

[5] Halim AM, Jacques JE. Rheumatic heart disease in Sudan. Br Heart J 1961; 23:383-5

[6] Hosmer, D. and Lemeshow, S. (2000).Applied logistic regression. $2^{\text {nd }}$ edition: John Wiley and Sons, New York.

[7] Khalil SI. El FatehZein, Gisouli D. Kazzam E, Rabeh R. Patterns of cardiovascular disease in Sudan. Sudan Medical Journal 1984; 20:25-3.

[8] Khalil, SI, Al Zain, AE. Heart failure in Sudan: Hospital Load and recent trends. Cardiovascular Therapeutics 2012; 30.

[9] Opie LH, Mayosi BM. Cardiovascular disease in sub-Saharan Africa. Africa. Pub Med Circulation. 2005; 112:3536-3540.

[10] Pampel, F. (2000). Logistic regression: A primer. Thousand Oaks, CA: Sage Publications.

[11] Suliman AA. Pattern of heart disease at AlShab Teaching Hospital; a decade into the new millennium. Sudan Med J 2011; 47:86-93.

[12] World Health Organization. The global burden of disease: 2004 update. Available at http://www.who.int/healthinfo/global_burden_disease/ 2004_report_update/en/index.html_ (accessed 2 December 2009) 